\title{
Flow in Left Atrium Using MR Fluid Motion Estimation
}

\author{
Kelvin K. L. Wong*a ${ }^{* a}$ Richard M. Kelso ${ }^{\mathrm{b}}$, Stephen M. Worthley ${ }^{\mathrm{c}}$, Prash Sanders ${ }^{\mathrm{c}}$, \\ Jagannath Mazumdar ${ }^{\mathrm{a}}$, Derek Abbott ${ }^{\mathrm{a}}$ \\ ${ }^{a}$ Centre for Biomedical Engineering and School of EEE, The University of Adelaide, SA5005; \\ ${ }^{\mathrm{b}} \mathrm{School}$ of Mechanical Engineering, The University of Adelaide, SA5005 \\ ${ }^{\mathrm{c} C}$ ardiovascular Research Centre and School of Medicine, The University of Adelaide, SA5005
}

\begin{abstract}
A recent development based on optical flow applied onto Fast Imaging in Steady State Free Precession (TrueFISP) magnetic resonance imaging is able to deliver good estimation of the flow profile in the human heart chamber. The examination of cardiac flow based on tracking of MR signals emitted by moving blood is able to give medical doctors insight into the flow patterns within the human heart using standard MRI procedure without specifically subjecting the patient to longer scan times using more dedicated scan protocols such as phase contrast MRI. Although MR fluid motion estimation has its limitations in terms of accurate flow mapping, the use of a comparatively quick scan procedure and computational post-processing gives satisfactory flow quantification and can assist in management of cardiac patients. In this study, we present flow in the left atria of five human subjects using MR fluid motion tracking. The measured flow shows that vortices exist within the atrium of heart. Although the scan is two-dimensional, we have produced multiple slices of flow maps in a spatial direction to show that the vortex exist in a three-dimensional space.
\end{abstract}

Keywords: MR fluid motion estimation, optical flow, left atrium, vortex

\section{INTRODUCTION}

Cardiac diseases are the leading cause of deaths in most developed countries. Various screening methods for heart defects exist in the medical industry. Some of the most common ones are electrocardiogram, echocardiography, and chest radiography. Other diagnostics reports can be based on cardiac magnetic resonance imaging and computed tomographic scans. Cardiac examination and management of patients remain the key priority in treatment of heart defects; however, the need for a concise insight of the defect is most necessary for diagnosis.

A good cardiac diagnostics system will have the ability to provide information about the heart that can be utilized for effective analysis depending on the nature of the diagnosis. For our study, we based the diagnosis on the analysis of flow maps that can be used to examine abnormal flows or channels that arises from the heart structural abnormalities. We examine the left atria of five patients with atrial septal defect (ASD) in order to understand the flow patterns of blood in abnormal heart. To achieve this objective, there is a need to measure and produce the visualization of flow within cardiac chambers for characterization and analysis.

The MR fluid motion estimation scheme that we proposed is used for flow measurement computationally on MR images for blood flow visualization. From the results produced, we can assess the nature of blood flow from MR imaging without modification of scanning protocol or additional procedure that is computationally exhaustive or time consuming. The application is able to give an insight of blood flow in the human heart using non-invasive means. Although the scheme is limited in terms of accuracy of motion estimation technically, it is still able to provide good qualitative information in the form of vector fields that can be used for flow analysis.

MR fluid motion tracking on MRI relies on the motion estimation of MR signals that dynamically shifts as the fluid is transported. This dynamic signal capture is typical of the standard True FISP MRI scan outputs. By concatenating a series of images pertaining to more than one time phase, the change in signal intensity can be detected and tracked to give vector flow field. For this, multi-resolution optical flow is applied onto every pair of MR images to produce the required flow maps.

*kelvin.wong@adelaide.edu.au; phone +61 08 8303-6296; fax +61 08 8303-4360;

Complex Systems II, edited by Derek Abbott, Tomaso Aste, Murray Batchelor, Robert Dewar,

Tiziana Di Matteo, Tony Guttmann, Proc. of SPIE Vol. 6802, 68021H, (2008)

$0277-786 \times / 08 / \$ 18 \cdot$ doi: $10.1117 / 12.787253$

Proc. of SPIE Vol. $680268021 \mathrm{H}-1$ 
A vorticity scheme based on summation of vectors surrounding every pixel coordinate throughout the whole image produces a vorticity map, and can be used to visualise the magnitude of rotation experienced by the blood in the heart chamber. Based on our implementation, we have successfully enabled the flow visualization of left atria with qualitative analysis of the vorticity in addition to the flow field. Examination of flow can be based on velocity vectors that are superimposed onto the vorticity map, and whose values can be represented using a color scale. The histogram for a flow phase is a plot of frequency of pixels corresponding to a scale of vorticity values throughout the map. This frequency plot is used to provide some indication for intensity of the degree of blood rotation within the cardiac chamber.

This paper is characterized by the following sections. Section 2 gives a brief literature review of the existing scanning protocols in cardiac flow mapping. Section 3 describes the methodologies and scan protocols for flow imaging in our case studies. The scan outputs of the experiment and analysis are provided in Section 4. In this set of results, we can observe the flow maps pertaining to left atria of one selected patient. Global analysis of the ASD study is also presented. Finally, a conclusion and suggestions for further application of MR fluid motion tracking is given in the last section.

\section{BACKGROUND}

\subsection{Ultrasound}

Ultrasound has been used widely in imaging cardiovascular flow in human for diagnosis. Doppler color flow imaging is now a widely accepted technique to measure subtle fluctuations in arteries and veins. Ultrasound images of flow are based on the Doppler Effect in which a series of pulses is transmitted to non-stationary blood to record echo signals from it. A color flow image or sonogram can be produced by recording the Doppler frequency shift.

Ultrasound techniques avoid the use of harmful radiation to the body, but produces relatively poor spatial resolution and often limited by the availability of a clear acoustic window between the external surface and imaged region. Moreover, it is difficult for ultrasound imaging to be performed on a cross-sectional plane of the chamber. Ultrasound scan is typically based on channel flow in the blood vessels of circulation system and not on a plane through the heart chamber that we are required to examine. As a result of this limitation, it is difficult for us to examine the existence of vortex in blood pool of the heart chamber.

\subsection{Magnetic Resonance Imaging}

Magnetic resonance imaging is the application of magnetic fields and usage of radiofrequency radiation to image difference tissues in the human body. The MR registration is based on the response of specific nuclei to the exposed radiofrequency energy and can be represented using an intensity-based image. The emitted signal can be encoded in such as way that the components that correspond to the spatial position of radiation emitting nuclei are used for construction of an image slice. An MRI scanner is effectively a device that creates a map of spin relaxation times of hydrogen nuclei within the tissue. This allows the physiology to be scanned at a planar section. MRI also allows multiple contiguous slices at various sectioning of the body to be scanned at various phases of one cardiac cycle. Therefore, cine-MRI can be presented at various phases continuously for motion analysis of cardiac structure.

\subsection{X-rays}

Radiographic techniques produce image films by registering the amount of X-ray through a human body. Variation in density of tissues results in different degrees of attenuation of X-ray photons within the physiology under exposure and gives the intensity contrast of tissues captured on film by casting a distinctive image of shadows on it. The use of ionizing radiation may harm the body if there is constant exposure. Due to the inability to register signals from localized regions, the scan is unable to produce an image registering a projection slice at specific depth through the body.

\subsection{Computed Tomography}

3D Computer Tomography (CT) creates a three dimensional visualization of the human body from a series of two dimensional X-ray scans taken about an axis of rotation. These images are synthesized into a model that allows the volume of data to be reformatted to present slices of structure within the human body at localized sections. Images of high temporal resolution can be registered using retrospective ECG gating. CT scans provide better soft tissue and bone contrast as compared to conventional X-rays and MRI. 


\section{METHODOLOGY}

\subsection{Selection of Imaging Modality for Blood Flow Tracking}

In our development, the objective is to map the flow of blood in human left atria. Imaging planar sections of the heart through the atria is one of the pre-requisite selection criteria for tracking vortical blood flow, since a vortex can be best characterized if sectional slice is taken orthogonal to the axis of rotation and presented in the plan view. Other views may not result in good qualitative characterization of the flow structure. Therefore, only spatially encoded signals from imaging modalities are to be utilized for blood flow imaging in the cardiac chamber especially. These scanning protocols allow imaging to be registered within the body at localized sections with specific depth and thickness.

Another selection criterion to be considered is that the imaging has to give quantifiable measures of blood flow at selectable positions of registration. Current imaging modalities such as phase contrast MRI and ultrasound are able to generate flow maps using colors to represent velocity magnitudes.

Finally, it is critical that the images can be produced in cine mode so that multiple phases of scan can be produced for continuous flow tracking throughout the entire cardiac cycle. Generally, retrospective ECG gating enables the imaging modality to develop images of high temporal resolution.

We have utilized TrueFISP MRI for vorticity tracking application in this paper. Although this imaging modality is unable to physically measure the flow dynamics of blood, we can still deduce flow patterns by assessing signal contrast representation of turbulence that moves along with blood flow. We considered a new method of computationally predicting blood movement for planar slices of scan by application of motion estimation algorithm on MR images pertaining to two or more consecutive phases. The details of the development are provided in the subsequent sections.

\subsection{TrueFISP Magnetic Resonance Imaging}

We examine the concepts of MR imaging at the simplistic level. An organic structure positioned within the central of an external magnetic field becomes partially magnetized itself with a magnitude of comparatively lower orders. The assemblage of hydrogen nuclei (protons) in the water molecules within the body can be perturbed with radiofrequency radiation. The nuclear spins will then realign with the magnetic field and in the process of doing so, emit radiofrequency $(\mathrm{RF})$ waves during this longitudinal relaxation period. Time $1\left(T_{l}\right)$ is defined as the duration for nuclear realignment and emission of RF signals that can be registered onto the MR images that we use for examination. Since the rate of emission of the RF waves is dependent on the type of material that contains the nuclei, different intensity of pixels representing the tissues can distinguish various anatomical structures.

Based on a similar type of magnetic field configuration, pulses of radio waves that have their magnetic moments perpendicular to the magnetic field applied can cause the hydrogen nuclei to have magnetic moment transverse to their original orientation. Realignment of nuclear spins after this transverse magnetization has a decay time constant labeled as Time $2\left(T_{2}\right)$ for this transverse relaxation. Likewise for tissue classification, the rate of decay is dependant upon the material nuclei, and therefore registers differently onto the MR image that is made up of pixels with varying intensity.

TrueFISP MRI is a modality capable of imaging cross-sections of cardiac structures with unsurpassed soft tissue contrast. It is one of the most popular medical imaging modality for registration of physiological properties of the heart and arteries. TrueFISP MRI combines both longitudinal and transverse magnetization ${ }^{3}$. It is characterized by a complex $T_{2} / T_{1}$-contrast configuration ${ }^{4}$ and refocuses all gradients over a repetition interval, thereby achieving fast imaging with high signal ${ }^{3}$.

\subsection{Asynchronous Precession of Hydrogen Nuclei in Turbulent Flow}

This section describes the theory governing the concept of void signal registration due to turbulence within fluid whose atomic nuclei have been aligned either parallel or anti-parallel to a powerful and uniform magnetic field. As the highenergy nuclei relax and realign, they emit energy with certain properties that are recorded to provide information about the medium. Image contrast is created by weighting the energy signal during realignment of the nuclear spins with the magnetic field. A signal image is generated as a result of this quantum mechanical activity. As the fluid is transported, the same signal from nuclei that is retained within the fluid follows the displacement. In a turbulent flow, the diffusion of magnetic moments occurs ${ }^{5}$. Protons in the hydrogen nuclei of molecules can be given a phase spin and the de-phasing (asynchronous precession) of spin due to turbulence in flow gives a low MR signal during imaging ${ }^{6}$. Therefore, there is a reduction in the signal registration onto the image. Effectively, diffusivity of spin protons at a point is represented by a 
reduction in signal intensity in the image ${ }^{5}$. Because the diffusivity follows the movement of the fluid in a channel, there exists an intensity change in the direction of flow. The intensity contrast of the diffusion in the image becomes greater as the speed of the fluid flow increases.

\subsection{Motion Estimation of MR-signals}

A series of MR images are taken temporally as the fluid is in motion. The velocity of dynamic fluid can be quantified in real time by computing the shift of intensities within the quantized regions of every prior and post images numerically. A velocity flow field can be constructed using a graphical plot as such and other fluid dynamics properties can be derived from the velocity flow measurement. From the results, the characteristics of the fluid flow can be analyzed using these properties.

We have developed a methodology for computationally determining the movement of fluid in a vessel based on motion estimation of the contrasting MR-signals. The motion of localized turbulence is influenced by the general flow globally. Motion estimation using multi-resolution optical flow technique is able to track the movement of the flow at various resolutions and resolve them to produce a global flow field in two dimensions. Therefore, we termed this approach as MR fluid motion estimation, as it is able to compute motion of MR imaged fluid.

Application of flow based on the use of motion estimation algorithm allows us to produce flow vectors over the region of analysis defined. The technique makes use of images from two subsequent phases to predict the flow field. Typically, cine MRI scanning results in a sequence of $N$ phases. Post-processing of the data from $(N-1)$ pairs of images gives a series of flow field displays for evaluation and analysis.

\subsection{Computational MR Fluid Motion Estimation}

A method of performing fluid motion tracking using TrueFISP MR images of non-stationary flow has been suggested recently ${ }^{1,2}$. In our approach, the optical flow algorithm which is a class of motion estimation methods is utilized. It generates flow vectors that correspond to the apparent motion of brightness or intensity patterns in the image ${ }^{8}$.

In our optical flow scheme, the pyramidal Lucas Kanade optical flow method ${ }^{9}$, which incorporates a multi-scale approach, has been applied to support large scale fluid motion and for improved accuracy. A top-down estimation of the flow by using an image pyramid is performed, with the apex representing the MR image at a coarse scale. Computational results from this level are passed to the next and this process is carried on based on the flow estimated at the preceding scale until the original scale is reached. We refer to the diagram in Fig. 1 to illustrate the computational aspect of pyramidal optical flow. We project the computed coarse-level flow field onto the next finer pyramid level and continue this process for each level of the pyramid until the finest pyramid level has been reached.

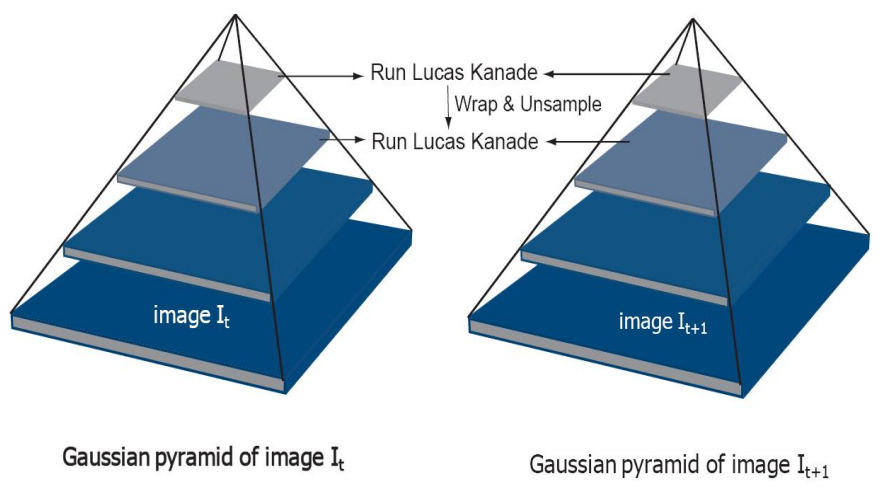

Fig. 1. Diagrammatic view of the pyramidal optical flow process. Each level in the pyramid is a sub-sampled version of the level below. In the first step the optical flow between the top level images is computed.

The accuracy of motion estimation critically depends on the magnitude of image motion. In fact, depending on the spatial image frequency, very large motions even may cause aliasing along the time frequency axis. For a fixed global velocity, spatial frequencies moving more than half of their period per frame cause temporal aliasing ${ }^{10}$.

For signal emitting nuclei motion that are represented by intensity pixels on MR images, the application of multiresolution optical flow scheme that predicts fluid motion based on grey-level constancy assumption (optical flow constraint). 


\subsection{Velocity and Vorticity Map Representations}

A dense velocity field (one velocity vector per pixel) is displayed and a vector averaging for sampling window resolution of 3 by 3 pixels is carried out. Scaling is carried out with respect to all the vectors present in the displayed phases of the cardiac cycle for ease of visualization and referencing. However, for analysis of dominant vortices, the flow values which appeared within each segmented vortex per slice have their magnitudes scaled independently. Positive values signify clockwise rotation (CW), whereas negative values represent counter-clockwise (CCW) motion of the blood. These may be represented by a color scale with maximum CW and CCW values represented by red and blue respectively (as observed in the figures showing characteristic flow in this paper).

\subsection{Flow Characteristics}

Statistical quantification of blood flow in the atria can be performed by examining the vorticity, shear strain and normal strain of the blood. These measures flow are computed from the velocity vectors in the motion field produced by the optical flow scheme ${ }^{4}$. Of course, other measures could also be calculated within the scope of the present technique. Based on the velocity field, with its $x$ and $y$ components as $V_{x}(i, j)$ and $V_{y}(i, j)$ respectively, about a point of interest located at $(i, j), N$ represents the number of layers of the contour within the sampling frame and $\Delta x$ and $\Delta y$ represent the horizontal and vertical distances between neighboring measurement points. The flow characteristics can then be numerically determined.

For vorticity $(\omega)$, the summation of vectors in the clockwise direction around a point of measurement results in the equation,

$$
\omega=\sum_{n=1}^{N}\left\{\frac{V_{x}(i+n, j)-V_{x}(i-n, j)}{2 n \Delta y}-\frac{V_{y}(i, j+n)-V_{y}(i, j-n)}{2 n \Delta x}\right\} .
$$

Shear strain $(\Phi)$ can be computed by the summation of vectors around a point of measurement, of which half were taken for the clockwise and the other half for counter clockwise directions, to give

$$
\Phi=\sum_{n=1}^{N}\left\{\frac{V_{x}(i+n, j)-V_{x}(i-n, j)}{2 n \Delta y}+\frac{V_{y}(i, j+n)+V_{y}(i, j-n)}{2 n \Delta x}\right\} .
$$

The normal strain ( $\Psi$ ) determines pressure experienced by the blood at local positions and this has some implications in strain of the bio-fluid. It is given by the summation of vectors across the boundaries of the contour as

$$
\Psi=\sum_{n=1}^{N}\left\{\frac{-V_{x}(i+n, j)+V_{x}(i-n, j)}{2 n \Delta y}+\frac{-V_{y}(i, j+n)+V_{y}(i, j-n)}{2 n \Delta x}\right\} .
$$

\section{EXPERIMENTS}

\subsection{Subjects for Case Study}

We performed MRI scans of five patients with atrial septal defects (ASD). The patients had an ASD closure. We select CMR image slices of the patient that corresponds to diastolic phases of the heart. We observed the vortices that are generated pre-septal occlusion at the selected phases. A region of interest with width of 120 pixels and height of 150 pixels is truncated from the selected slice showing the sectioning of the right and left atria, where the ASD is most apparent. This is to be displayed for qualitative examination.

\subsection{MRI Scan Procedure}

For this research, CMR imaging is performed using a Siemens Sonata, 1.5 Tesla, model - Syngo MR 2004A scanner with Numaris - 4, Series No: 21609 software. Steady-state free precession cine-MR imaging was performed using contiguous slices in short axis views through the ASD. All images were acquired with retrospective gating and 25 phases (from $T=1$ to 25 ) for each slice are obtained. The images are standardized to sizes of $156 \times 192$ pixels in order to maintain consistency for comparison during computation and analysis. 


\subsection{Data Analysis}

Based on the measured velocity field, a sampling frame of 21 by 21 windows is used for the vorticity and strain calculations. This corresponds to 10 layers of neighboring contours. Smoothing of vector components is carried out within a 3 by 3 pixel window.

Statistical quantification of blood flow in the left atrium, is performed by examining the vorticity, shear strain, and normal strain of the blood. These measures are computed from velocity vectors in the motion field produced by the optical flow scheme.

Normalization is a means of standardizing the total count of pixels within a segmented region of interest (the examined atrium) and this is equal to sum of all bin counts of the histogram. Normalization of the histograms is performed by setting the sum of all counts in the bins to 3500 . The total counts correspond to the number of pixels representing the atrium per slice. The number of bins is set at 35 .

\section{RESULTS}

\subsection{Details of Analysis}

The observation of vortices and statistical analyses are performed for the left atria and displayed on the left and the right respectively in all of the figures depicting the flow results. The global estimation of flow velocity vector fields over the whole image provides useful information on the presence of vortices within the atrium that can be used to assess the vorticity. The magnitude of vortical flow can be determined from the mean of vorticity histogram, $\bar{\omega}$, and the shear strain $\bar{\Phi}$ and normal strain, $\bar{\Psi}$ that are associated with it. The results using MR images at a particular slice for all left atria can be shown in appendix A.

\subsection{Vorticity Variation in Left Atrium}

The flow patterns of blood within the left atria of ASD patients are presented in Fig. 2. The images pertaining to phases that show the most obvious vortices are shown. The histograms based on counts of vorticity values within the segmented chamber illustrate the spread of the degree of blood rotation.

It is to be noted that the velocity magnitudes are not calibrated against realistic measurements of blood flow physically and therefore is limited in terms of accurate quantification with standard international metric units. However, the computational values that the application generates provide good quantification of relative vorticity measurements that can be used to assess different levels of vorticity for comparison before and after surgical intervention. Based on this arrangement, the development of this technique is still able to contribute diagnostics value to the study of ASD patients.

Case study reports depicting the quantification of blood flow within the left atrium are built for clinical assessment after MR scans. The advantage of this cardiac health assessment system is the speed of preparation and the redundancy of modifying scan modes during MR imaging.

Clinical verification of ASD patient has been successfully accomplished previously, but it has been based on the right atria only ${ }^{7}$. In this study, case report generations of the atrial flow for a sample ASD patient are presented. Reliability in testing patients for cardiac abnormalities is the key work at this stage.

Generally, at a particular time instance, if the vector field demonstrated that most regions of blood in the atrium are stagnant or experiencing a laminar flow, the mean vorticity should be close to zero. In the case of the abnormal heart, it is likely that the mean vorticity and strain will shift away from zero, and broaden the distribution of the histogram. The display of information using histograms of vorticity, shear and normal strains can provide a clear overview of the swirl and stress present within the blood flow. Therefore, as demonstrated, the flow vorticity, shear strain and normal strain counts for pre-operation are more significant than those for the post-operation. This is evidence that the methodology is successful in assessing the relative intensity of vorticity before and after insertion of the septal occluder. 

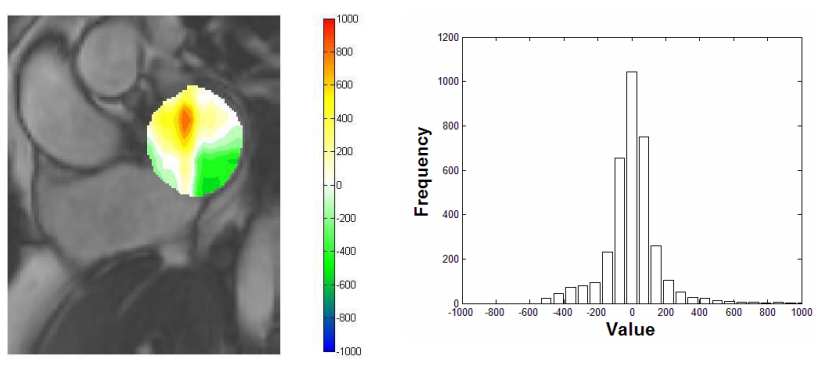

(i) $T=15 \quad \bar{\omega}=-0.44, \bar{\Phi}=-7.42, \bar{\Psi}=9.34$
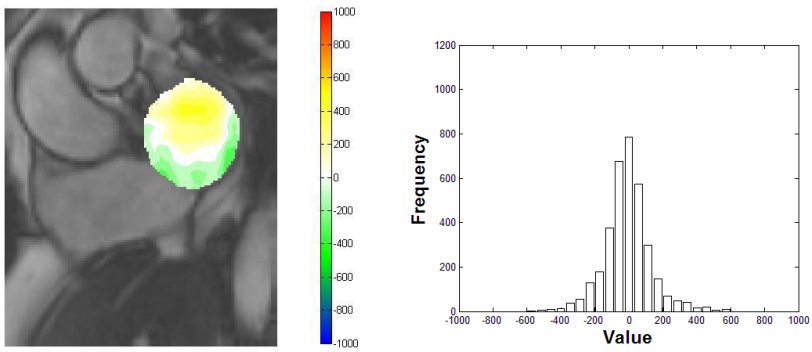

(ii) $T=16 \quad \bar{\omega}=-7.09, \bar{\Phi}=4.49, \bar{\Psi}=14.82$
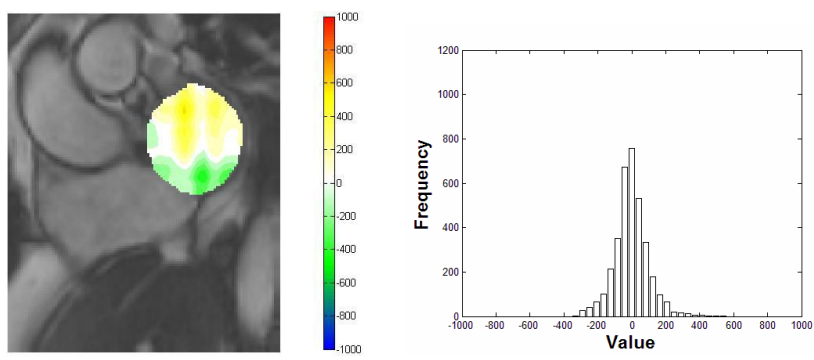

(iii) $T=17 \quad \bar{\omega}=-2.35, \bar{\Phi}=-22, \bar{\Psi}=5.06$
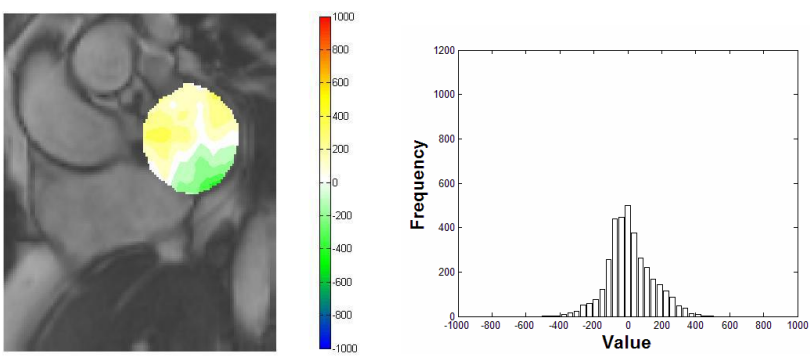

(iv) $T=18 \quad \bar{\omega}=11.43, \bar{\Phi}=39.13, \bar{\Psi}=-5.51$

(a) Pre-ASD 

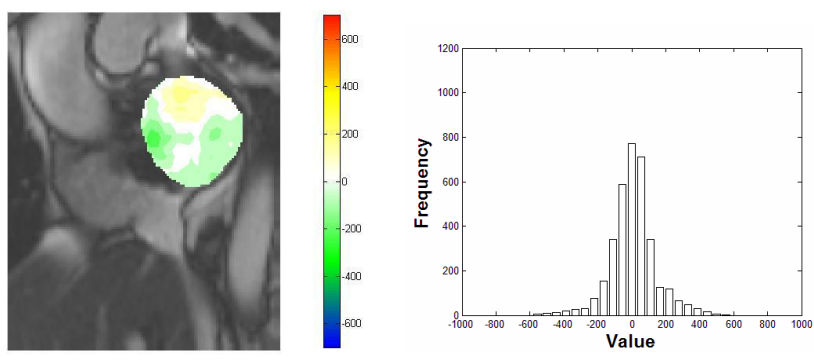

(i) $T=15 \quad \bar{\omega}=8.96, \bar{\Phi}=70.44, \bar{\Psi}=21.04$
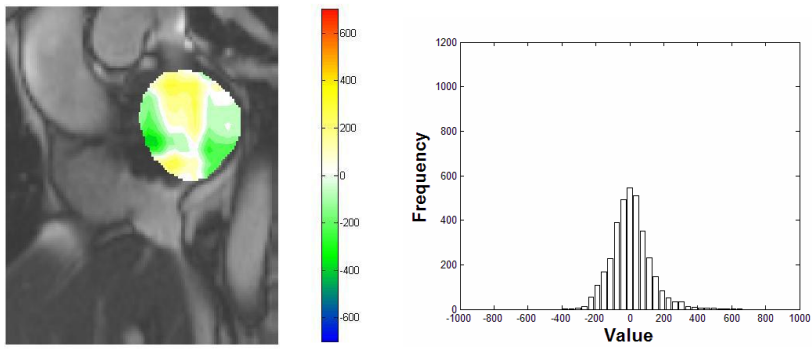

(ii) $T=16 \quad \bar{\omega}=6.02, \bar{\Phi}=29.89, \bar{\Psi}=-7.38$
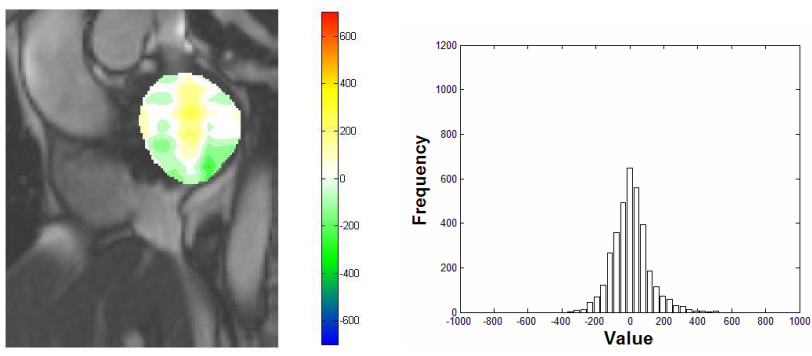

(iii) $T=17 \quad \bar{\omega}=5.38, \bar{\Phi}=24.88, \bar{\Psi}=2.23$
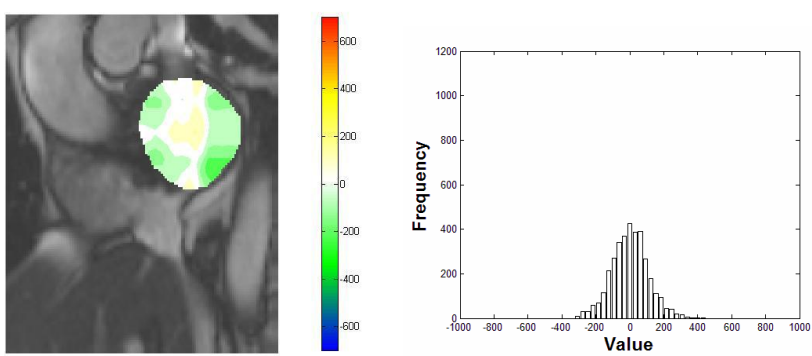

(iv) $T=18 \quad \bar{\omega}=1.38, \bar{\Phi}=45.3, \bar{\Psi}=16.28$

\section{(b) Post-ASD}

Fig. 2. Vorticity visualization from CMR scans of segmented atrium at 4 phases $(T=15,16,17,18)$ of cardiac cycle. The flow characteristic is shown for vorticity, normal strain, and shear strain starting from top to bottom. 


\subsection{Analysis of Atrial Septal Defect Patients}

The variation in the level of these parameters at each phase can be used to determine the degree of abnormality in the cardiac structure. A sudden change in value of the flow parameter depicts a flow event at a particular time phase. We are able to show in our study that the vorticity in left atrium for pre-ASD is consistently higher than for post-ASD. The research shows that we are able to use MR fluid motion tracking to find a difference in flow patterns before and after surgical intervention. The technique can also be used to analyze shear strain and normal strain of blood within a human cardiac chamber.

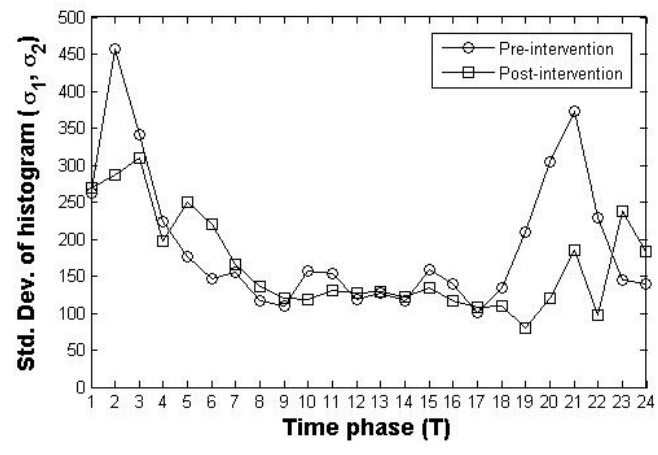

(a) Vorticity

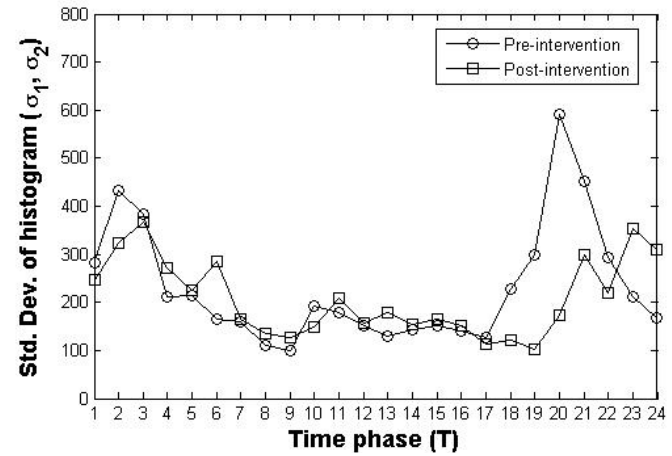

(b) Shear Strain

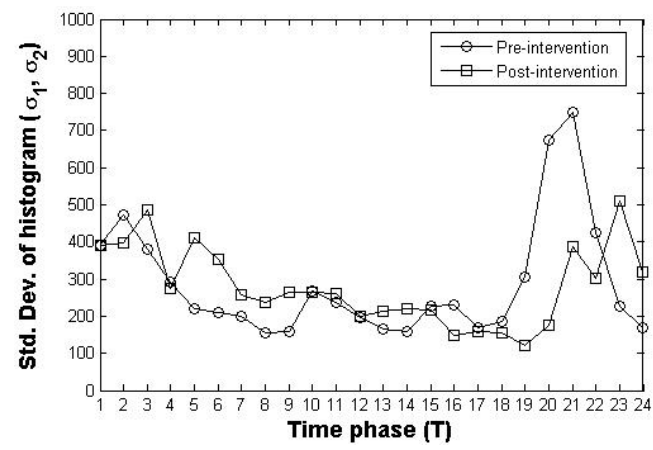

(c) Normal Strain

Fig. 3. Sample case study report showing the relevant statistical information that is to be displayed and analyzed by cardiologists. Variation of vorticity variance for a sample patient, with three flow characteristics: (a) vorticity, (b) shear strain, (c) normal strain of pre- and post-ASD closure are displayed from phase 1 to 24 respectively.

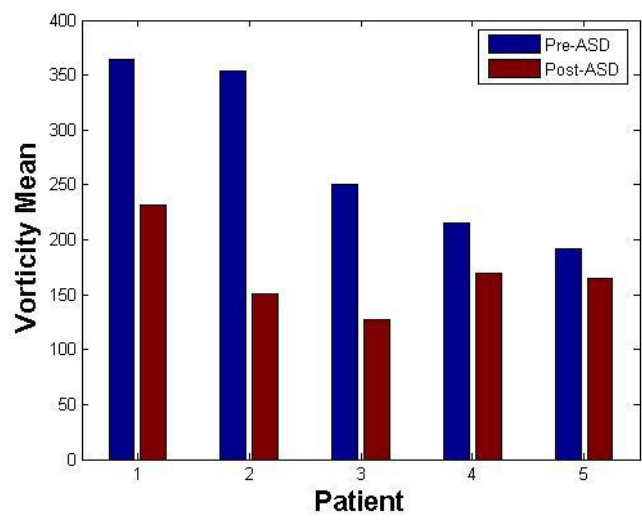

Fig. 4. The mean of vorticity standard deviations for every patient is computed. The graph demonstrates that the vorticity is smaller after septal occlusion. The results serve as evidence for the proposed framework to be used as a diagnosis for ASD or assessment of patients after ASD-closure. 


\section{CONCLUSION}

We have obtained results for a heart with a septal defect (prior and post septal occlusion). The study has showed that, based on the calculations of vorticity, shear strain and normal strain, it is possible to detect the intensity of vorticity and therefore determine the degree of septal defect, and provide some indication of the success of the septal occluder insertion. The application may also be used to examine the growth of cardiac tissue into the mesh of the occluder. As it provides quantitative as well as qualitative data for this flow, the scheme can be of great benefit to surgeons for assessing their surgical success.

The MR fluid motion tracking method has been tested successfully for an abnormal pathology and a more quantitative diagnosis to add to the qualitative information has been obtained. The application may potentially be incorporated into MRI visualization modeling and display for such a purpose.

\section{REFERENCES}

$1 \quad$ K. K. L. Wong, P. Kuklik, S. G. Worthley and P. Sanders, "Flow Analysis," International (PCT) Patent Application PCT/AU/2007/000827, (PCT application filed 15th June 2007).

2 K. K. L. Wong, P. Molaee, P. Kuklik, R. M. Kelso, S. G. Worthley, P. Sanders, J. Mazumdar, and D. Abbott, "Motion estimation of vortical blood flow within the right atrium in a patient with atrial septal defect," 2007 IEEE/ICME Int. Conf. on Complex Med. Eng., Beijing China, 868-875 (May 2007).

3 Q. Chen, P. Storey, D. Levine, W. Li, and R. Edelman, "A Breath-hold Three Dimensional True FISP Sequence for Abdominal MRI,” Proc. Intl. Soc. Mag. Reson. Med. 9, (2001).

4 C. U. Herborn, F. M. Vogt, J. F. Debatin and S. G. Ruem, “Assessment of pancreatic lesions by MRI: TrueFiSP versus HASTE," Proc. Intl. Soc. Mag. Reson. Med. 10, (2002).

5 D. O. Kuethe, "Measuring distributions of diffusivity in turbulent fluids with magnetic-resonance imaging," Phys. Rev. A 40, 4542 - 4551 (1989).

6 M. A. Lawson, "Cardiovascular imaging in the new millennium," BUMC Proceedings 12, 115-120 (1999).

7 K. K. L. Wong, P. Kuklik, R. M. Kelso, S. G. Worthley, P. Sanders, J. Mazumdar and D. Abbott, "Blood flow assessment in a heart with septal defect based on optical flow analysis of magnetic resonance images," Proc. SPIE Biomed. Appl. Micro- \& Nanoeng. III, 6416, Adelaide Australia, Art. No. 64160L (Dec 2006).

8 B. K. P. Horn, and B. G. Schunck, "Determining optical-flow," Artificial Intelligence 17(1-3), 185-203 (1981).

9 J. Y. Bouguet, "Pyramidal implementation of the Lucas Kanade feature tracker," Technical Report, OpenCV Documentation, Microprocessor Research Labs, Intel Corp, (2000).

10 P. Ruhnau, T. Kohlberger, H. Nobach and C. Schnorr, "Variational optical flow estimation for particle image velocimetry," Exp. Fluids 38(1), 21-32 (2005). 